\title{
Analysis of weapon systems protecting military camps against mortar fire
}

\author{
M. Graswald, I. Shaydurov \& H. Rothe \\ Helmut-Schmidt-University, Hamburg, Germany
}

\begin{abstract}
The protection of military camps that are subject to attacks by rockets, artillery projectiles, or mortar grenades (RAM) is currently in the spotlight of Western nations involved in overseas missions. Due to its worldwide distribution and good combat properties for guerilla warfare, mortars are likely to be applied and therefore selected as RAM threat in this paper. For this case, camp safety shall be ensured by an early warning system and an air defense weapon system using $35 \mathrm{~mm}$ Ahead ammunition. For both systems, a key to success is the accuracy of the sensors used, namely the radar. Therefore after providing the mathematical background, simulations are conducted in order to find the variations of the hit point of mortar shells. Moreover, the consumption of Ahead ammunition to engage and destroy typical $82 \mathrm{~mm}$ grenades is estimated. From these results, the suitability of present radar sensors and air defense systems and the technical requirements of future weapon systems can be derived.

Keywords: rockets artillery mortar (RAM), $35 \mathrm{~mm}$ Ahead ammunition, radar sensor, exterior ballistics, terminal ballistics, probability calculation, error propagation, circular error probability, ammunition consumption.
\end{abstract}

\section{Introduction}

Accomplished missions of the Western military e.g. in Iraq or Afghanistan in the recent past have shown that the safety of military camps is not sufficient. This is because suitable weapon systems protecting the military installations against this new asymmetric threat do currently not exist. Furthermore, these RAM attacks by guerillas or terrorists are frequently undertaken from urban area and therefore, the rules of engagement do usually not allow counterstrikes. Primarily, mortar grenades of 60 and $82 \mathrm{~mm}$ caliber and unguided $107 \mathrm{~mm}$ rockets are distributed 
all over the world in great quantities and relatively easy to obtain and thus chosen for attacks.

This study concentrates on $82 \mathrm{~mm}$ mortar grenades as a typical example for the RAM threat. Two different approaches to increase the camp safety are investigated and requirements will be given for:

- an early warning system that alerts soldiers depending on the predicted point of impact of the mortar shell,

- an air defense system that intercepts the shells in a safe distance with Ahead sub-projectiles forming a fragment cone, fig. 1.

\section{Ballistic model}

A mathematical model is adopted estimating the ballistic coefficient of the grenade from four radar tracks of its flight path. As a result, it allows the iterative calculation of the trajectory from an averaged radar location to either the firing or the hit point.

\subsection{Exterior ballistics of mortar grenades}

Since the mortar grenades considered are arrow-stabilized and fired on short distances up to approximately $8 \mathrm{~km}$, the 2-DOF equations of motion are derived from Newton's second law treating the grenade as point mass and incorporating only gravitation and air drag as external forces. This leads to the system of differential equations given in a path-dependant form for a right-handed frame of reference shown in fig. 2 :

$$
\begin{aligned}
v_{x}^{\prime}=\frac{\mathrm{d} v_{x}}{\mathrm{~d} x}=-c_{2}(M a) v(x) & \text { (1a) } & y^{\prime}=\frac{\mathrm{d} y}{\mathrm{~d} x}=p(x) \\
p^{\prime}=\frac{\mathrm{d} p}{\mathrm{~d} x}=-\frac{g}{v_{x}(x)^{2}} & \text { (1b) } & t^{\prime}=\frac{\mathrm{d} t}{\mathrm{~d} x}=\frac{1}{v_{x}(x)}
\end{aligned}
$$

where $p=\tan \theta=v_{y} / v_{x}, v=v_{x} \sqrt{1+p^{2}}, v_{x}-x$-component of velocity, $v_{y}$ - $y$-component of velocity, $g-$ acceleration due to gravity $\left(g=9.81 \mathrm{~m} / \mathrm{s}^{2}\right), t-$ computed time of flight, $\theta$ - quadrant elevation, $c_{2}(M a)$ - coefficient of air drag depending on Mach's number.

\subsection{Trajectory determination by radar measurements}

A well-known procedure to experimentally determine the projectile's air drag is adopted to estimate the ballistic coefficient [1]. This coefficient is an essential input parameter solving eqns. (1) numerically in order to calculate the trajectory. It is iteratively computed starting at point B determined by radar measurements to the hit point, see fig. 3 .

To estimate the ballistic coefficient it is assumed that the velocity depending part of the air drag is known by an experimentally determined reference function 


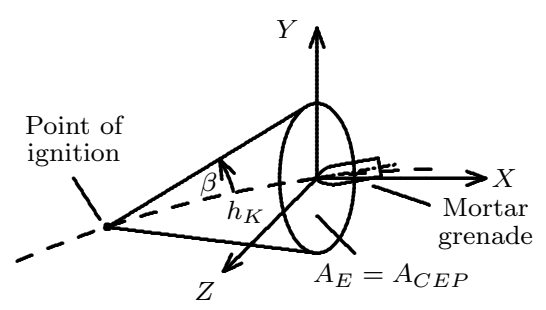

Figure 1: Fragment cone.

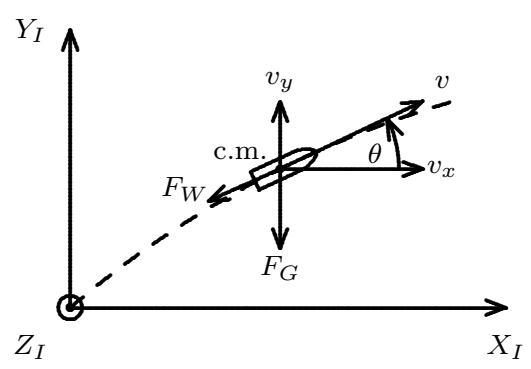

Figure 2: Inertial reference frame.

and the part depending on the atmosphere is considered as constant. Therefore, the term that depends on the projectile itself needs to be determined and is called ballistic coefficient $c$.

The drag coefficient $c_{2}$ ( $c$ can simply be derived from it) is determined by the drag function $F_{W}=\frac{\rho}{2} c_{W} v^{2} A$, which corresponds to the difference in kinetic energy in $\mathrm{A}$ and $\mathrm{B}$ and refers to the distance $x_{A B}$ :

$$
c_{2}=\frac{a_{W}}{v_{m}^{2}},
$$

where $a_{W}=\frac{1}{2}\left(v_{x_{A}}^{2}-v_{x_{B}}^{2}\right) / x_{A B}$ is the deceleration and $v_{m}=\frac{1}{2}\left(v_{x_{A}}+v_{x_{B}}\right)$ is the averaged horizontal component of the projectile's velocity. The horizontal distance of the averaged radar locations $\mathrm{A}$ and $\mathrm{B}$ is determined geometrically by $x_{A B}=\sqrt{\left(x_{B}-x_{A}\right)^{2}+\left(z_{B}-z_{A}\right)^{2}}$.

The velocities and coordinates in $x$ and $z$ at the locations A and B are calculated by two tracks of a monopulse radar, respectively. Due to the specific form of eqns. (1), only horizontal components of the velocity and the horizontal distance between $\mathrm{A}$ and $\mathrm{B}$ are required. In another step, these radar tracks $x_{i}, y_{i}, z_{i}(i=$ $1 \ldots 4)$ are linked to measuring quantities of radar azimuth $\alpha$, elevation $\varepsilon$, and time $t$.

\section{Error propagation}

The methods of error propagation are applied to the model in Section 2 in order to find variations of the point of impact depending on the sensor accuracy, i. e. variations in range, deflection, and the circular error probability (CEP). All systematic measuring errors shall be eliminated by calibration or adjustment. Thus, the measurements of the radar azimuth, elevation and time are subject to random errors. It is assumed that these random errors are normally distributed with $\mu=0$ as mean value and the standard deviations $\sigma_{\alpha}, \sigma_{\varepsilon}$, and $\sigma_{t}$ are given by the measuring devices. For a radar with rotating antenna its angular velocity $\omega$ is also subject to errors with $\sigma_{\omega}$ as standard deviation. 


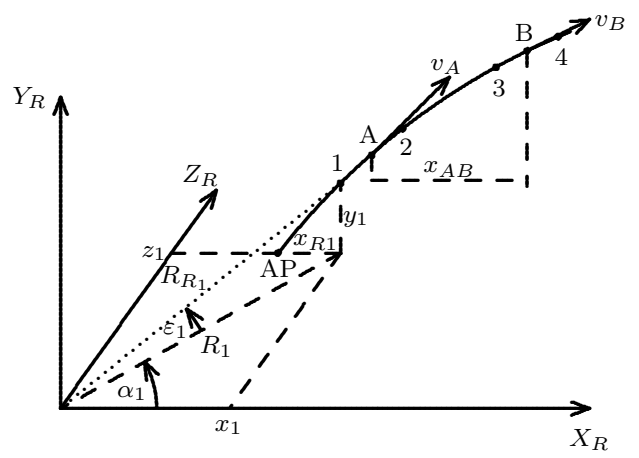

Figure 3: Radar reference frame with trajectory of mortar shell.

\subsection{Variations of the predicted point of impact}

As described, the ballistic coefficient $c$ is used to determine the trajectory and point of impact. Hence, errors of the radar measurements are propagated by the ballistic coefficient to the hit point and determine its desired variation. In order to calculate the variation in range, the standard deviation of the ballistic coefficient $\sigma_{c}$ is computed with the random errors of azimuth, elevation, and time by applying the laws of propagation of uncertainties:

$$
\sigma_{c}=\sqrt{\left(\frac{\partial c}{\partial x_{A B}}\right)^{2} \sigma_{x_{A B}}^{2}+\left(\frac{\partial c}{\partial v_{x_{A}}}\right)^{2} \sigma_{v_{x_{A}}}^{2}+\left(\frac{\partial c}{\partial v_{x_{B}}}\right)^{2} \sigma_{v_{x_{B}}}^{2}}
$$

where $\sigma_{x_{A B}}, \sigma_{v_{x_{A}}}$, and $\sigma_{v_{x_{B}}}$ are computed from given $\sigma_{\alpha}$ and $\sigma_{t}$ :

$$
\sigma_{x_{A B}}=\sqrt{\sum_{j=1}^{4}\left(\frac{\partial x_{A B}}{\partial \alpha_{j}}\right)^{2} \sigma_{\alpha}^{2}}, \quad \sigma_{v_{x_{A}}}=\sqrt{\sum_{j=1}^{4}\left(\frac{\partial v_{x_{A}}}{\partial \alpha_{j}}\right)^{2} \sigma_{\alpha}^{2}+\sum_{k=1}^{2}\left(\frac{\partial v_{x_{A}}}{\partial t_{k}}\right)^{2} \sigma_{t}^{2}},
$$

and

$$
\sigma_{v_{x_{B}}}=\sqrt{\sum_{j=1}^{4}\left(\frac{\partial v_{x_{B}}}{\partial \alpha_{j}}\right)^{2} \sigma_{\alpha}^{2}+\sum_{k=3}^{4}\left(\frac{\partial v_{x_{B}}}{\partial t_{k}}\right)^{2} \sigma_{t}^{2}} .
$$

The deviation of the angular velocity can be easily calculated with $\sigma_{t}$

$$
\sigma_{\omega}=\frac{2 \pi}{t^{2}} \sigma_{t}=\frac{\omega^{2}}{2 \pi} \sigma_{t}
$$

Subsequently, the approach of varying failure parameters can be used to calculate e.g. $N=1000$ normally distributed random quantities of the ballistic coefficient $c_{i}(i=1 \ldots N)$ with the mean value $\mu_{c}$ and standard deviation $\sigma_{c}$. These quantities are taken to determine the hit points $x_{i}$ by solving the system of differential equations (1) numerically. 
In order to compute the variation in range with these $x$-coordinates of the hit point, the averaged quadratic deflection $\mu$ is determined [1]

$$
\mu=\sqrt{\frac{\sum_{i=1}^{N} \lambda_{i}^{2}}{N-1}}
$$

where $\lambda_{i}=x_{i}-x_{m}$ is the so-called apparent error and $x_{m}=\frac{1}{N} \sum_{i=1}^{N} x_{i}$ is an averaged point of impact.

Given $\mu$ and the $u$-quantile of the normal distribution, the variation in range of the hit point $l_{P}$ at $P \%$ confidence level (C. L.) is calculated by [1]

$$
l_{P}=2 u \mu=2 P E_{R} .
$$

With the given errors of time and azimuth of the radar sensor, the variation in deflection is directly calculated based on the locating geometry $z_{i}=R_{i} \sin \alpha_{i}$. Considering the worst case, the standard deviation $\sigma_{z}=$ $\sqrt{\sin ^{2} \alpha_{i} \sigma_{R}^{2}+R_{i}^{2} \cos ^{2} \alpha_{i} \sigma_{\alpha}^{2}}$ reaches its maximum value for $\alpha_{i}=\frac{\pi}{2}$ and can be reduced to $\sigma_{z}=\sigma_{R}$. The range error of a radar is determined with the speed of light $c_{0}$ by $\sigma_{R}=\frac{c_{0}}{2} \sigma_{t}$.

Similar to eqn. (6), the variation in deflection $b_{P}$ is determined by

$$
b_{P}=2 u \sigma_{z}=2 P E_{D} .
$$

As final result, the CEP of the predicted hit point is determined with the variations in range and deflection. Gilles [2] introduces a procedure to compute the CEP on the desired C. L. with the standard deviations in $x$ and $z$ as well as the belonging covariance $\operatorname{cov}(x, z)$. As input parameters, the $x$-coordinates are already calculated, while the $z$-coordinates are $N=1000$ normally distributed random quantities generated with $\mu_{z}=0$ and $\sigma_{z}=\sigma_{R}$.

\subsection{CEP simulations}

For the following simulations, the commissioned radar systems MWRL-SWK (a Russian air surveillance radar for airports; $\sigma_{\alpha}=0.033 \mathrm{deg}, \sigma_{R}=10 \mathrm{~m}$, in RBS mode) and COBRA (a counter battery radar by Germany, France, and UK; $\sigma_{\alpha}=\sigma_{R} / R=0.191 \mathrm{deg}, \sigma_{R}=50 \mathrm{~m}$, system specification) may serve as typical examples (all radar and ammunition data throughout this paper are public domain).

An $82 \mathrm{~mm}$-grenade with propellant charge O-832, No. 6 launched with M1937 is chosen as RAM threat with the data $v_{0}=211 \mathrm{~m} / \mathrm{s}, c_{n o m}=2.11 \mathrm{~m}^{2} / \mathrm{kg}$, and $m=3.31 \mathrm{~kg}$.

Furthermore, the following data is given:

$$
\begin{aligned}
\omega & =90 \mathrm{deg} / \mathrm{s}, \alpha_{1}=145 \mathrm{deg}(\text { MWRL-SWK); } \\
\alpha_{1} & =145 \mathrm{deg}, \alpha_{2}=132,7 \mathrm{deg}, \alpha_{3}=114,6 \mathrm{deg}, \alpha_{4}=91,2 \operatorname{deg}(\text { COBRA }) ; \\
x_{A P} & =-2040 \mathrm{~m}, z_{A P}=1000 \mathrm{~m} .
\end{aligned}
$$


The general simulation results are shown in fig. 4, while the data for the selected radar sensors are tabulated in tab. 1 and integrated in fig. 4 for confidence levels of $50 \%, 90 \%$, and $99 \%$. Designing an early warning system for military camps, the estimation of $C E P=f\left(\sigma_{\alpha}=\sigma_{\varepsilon}, \sigma_{t}\right)$ allows the definition of the edge length of plane squares in order to enable specific areal warnings to soldiers. Since a clear threat direction is usually unknown, the camp shall be divided into squares with its edge length given by the CEP

$$
l_{P}=\lceil 2 C E P\rceil .
$$

For a very precise radar system like MWRL-SWK, its edge length is already $46 \mathrm{~m}$ at $50 \%$ C. L. and $98 \mathrm{~m}$ for $90 \%$ C. L. These distances cannot be covered in such short advance warning times of roughly $10 \mathrm{~s}$. It also becomes clear that the COBRA radar is insufficient for this task.

Table 1: Results of simulations for selected radar systems.

\begin{tabular}{|l||c|c||c|c|c|}
\hline \multicolumn{1}{|c||}{} & $c_{\text {cal. }}$ in $\mathrm{m}^{2} / \mathrm{kg}$ & $\sigma_{c}$ in $\mathrm{m}^{2} / \mathrm{kg}$ & \multicolumn{3}{c|}{$C E P$ in $\mathrm{m}$} \\
\hline C. L. in $\%$ & - & 68.3 & 50.0 & 90.0 & 99.0 \\
\hline \hline MWRL-SWK & 2.187 & 0.070 & 22.6 & 48.7 & 75.1 \\
\hline COBRA & 2.187 & 0.402 & 134.5 & 305.2 & 471.7 \\
\hline
\end{tabular}

\section{Ammunition consumption calculations}

Intercepting a RAM target successfully requires fulfilling two conditions: at first, the kinetic energy of the sub-projectiles is sufficient to destroy the mortar grenade and secondly, at least one pellet hits the shell. For this purpose, $35 \mathrm{~mm}$ x 228 Ahead ammunition is examined consisting of $N_{f}=152$ sub-projectiles and featuring fuze programming. Since the attempt of deflecting the grenade from its trajectory with the momentum of a sub-projectile is practically not promising due to the mass difference of factor $10^{2}$, this study concentrates on the physical destruction of the mortar grenade.

\subsection{Minimum energy for destruction of mortar grenades}

The minimum energy to destruct a grenade is compounded by the kinetic energy to penetrate the shell and the potential energy to activate the explosive. At first, the well-known formula of de Marre [3] is resolved for the desired minimum impact velocity $v_{\min }$ of an sub-projectile in order to penetrate a armored steel plate with thickness $D$ (in dm)

$$
v_{\min }(\gamma)=\frac{B d_{f}^{0.75} D^{0.7}}{m_{f}^{0.5} \cos ^{n} \gamma}
$$


Table 2: Minimum energy destructing typical Russian mortar grenades.

\begin{tabular}{|c|c||c|c|c|c|c|}
\hline \multicolumn{2}{|c||}{ caliber $d_{M}$ in $\mathrm{mm}$} & 82 & 107 & 120 & 160 & 240 \\
\hline \hline \multicolumn{2}{|c||}{$D_{\max }$ in $\mathrm{mm}$} & 10.2 & 16.5 & 19.1 & 21.8 & 39.3 \\
\hline$E_{\min }$ in J & $\gamma=0 \mathrm{deg}$ & 1160 & 2257 & 2756 & 3335 & 7563 \\
\hline$E_{\min }$ in $\mathrm{J}$ & $\gamma=30 \mathrm{deg}$ & 1778 & 3467 & 4236 & 5126 & 11636 \\
\hline
\end{tabular}

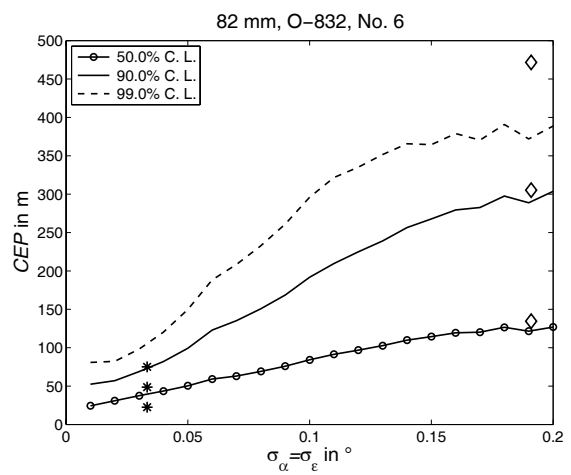

Figure 4: $C E P=f\left(\sigma_{\alpha}\right), \sigma_{t}=200 \mathrm{~ns}$ (*-MWRL, $\diamond-$ COBRA).

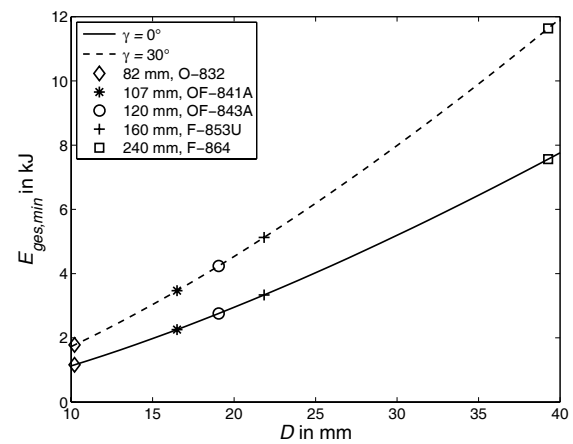

Figure 5: $E_{\min }=f(D, \gamma)$.

where $d_{f}$ - caliber of sub-projectile $\left(d_{f}=5.810^{-2} \mathrm{dm}\right.$, estimated), $m_{f}-$ subprojectile's mass $\left(m_{f}=3.310^{-3} \mathrm{~kg}\right), B$ - empirical factor $(B=2000$, averaged value), $\gamma-$ angle of impact (measured from plate's normal) and $n=1.5$ [3]. For this case, the maximum thickness of an $82 \mathrm{~mm}$ shell is set for $D$.

The energy to ignite the grenade's explosive is calculated with the impact sensitivity for TNT $m h=1.5 \mathrm{~kg} \mathrm{~m}$ and the acceleration due to gravity. Finally, the overall minimum energy is

$$
E_{\min }(\gamma)=\frac{m_{f}}{2} v_{\min }^{2}(\gamma)+m h g
$$

and is shown for several Russian mortar grenades and impact angles of $\gamma=$ 0 deg , 30 deg in fig. 5 as well as tab. 2.

Ideally, the internal energy $E_{i}$ provided through an rigid body impact of the sub-projectile with the grenade needs to be greater than the minimum energy $E_{\text {min }}=f\left(d_{M}, \gamma\right)$ given in tab. 2. Due to the small amount of HE, the subprojectiles are not accelerated after ignition. Therefore, the velocity of a subprojectile is calculated with the velocity of the Ahead projectile at time of ignition and decreased on its flight path because of air drag. 


\subsection{Kill probability}

In order to obtain a high kinetic energy for destruction, a frontal attack by an Ahead sub-projectile is considered as ideal case due to the high velocity difference of the sub-projectile and the grenade. Hence, the target area $A_{T}$ is calculated with the caliber radius of the shell. However, the chance to hit the fuze of the grenade in order to ignite it is not considered in this paper.

Calculating the hit probability of an sub-projectile at first, it is assumed

- the cannon is calibrated $\left(\mu_{y}=\mu_{z}=0\right)$,

- the ballistic deviation of cannon and ammunition is $\sigma_{y}=\sigma_{z}=\sigma_{b}$,

- the hit pattern in $Y$ and $Z$ are regarded as independent $(\rho=0)$.

Thus, the probability $p_{H F}$ to hit a circle area in a $Y$-Z-plane with radius $R_{M}$ with a single sub-projectile is based on the normal distribution [3]

$$
p_{H F}=1-e^{-\frac{R_{M}^{2}}{2\left(u \sigma_{b}\right)^{2}}} .
$$

Since the target has also a dimension in the $X-Y$-plane, the hit probability $p_{H t}$ in this plane is determined with the Gaussian error function and the relative target length $x_{r e l}=l_{M} / \sigma_{x}$ applying the distribution function $\Phi$ of the normal distribution

$$
p_{H t}=\operatorname{erf}(x)=2 \Phi(\sqrt{2} x)-\frac{1}{2}
$$

where $x$ is the input parameter determined as follows [1]:

$$
x=\frac{u}{\sqrt{2}} \frac{l_{M}}{\sigma_{x}}
$$

where $l_{M}$ is the grenade's length and the deviation $\sigma_{x}=\sqrt{\sigma_{x R}^{2}+\sigma_{x G}^{2}}$ consists of the converted time deviation of the radar $\sigma_{x R}=\frac{c_{0}}{2} \sigma_{t R}$ and the converted time resolution of the fuze $\sigma_{x G}=v_{G} \sigma_{t G}\left(v_{G}-\right.$ velocity of Ahead projectile). Finally, the desired hit probability of a single sub-projectile with the above probabilities being independently is

$$
p_{H}=p_{H F} p_{H t} .
$$

A single effective sub-projectile shall be able to destruct a grenade. Thus, the destruction probability is calculated with the internal energy $E_{i}$ [3]

$$
p_{k \mid H}=1-e^{-\frac{E_{i}}{E_{0}}},
$$

where $E_{0}$ is given by the minimum energy $E_{\min }(\gamma)$ at $\gamma=0 \mathrm{deg}$.

In conclusion, the kill probability of a single sub-projectile is

$$
p_{K}=p_{H} p_{K \mid H} .
$$



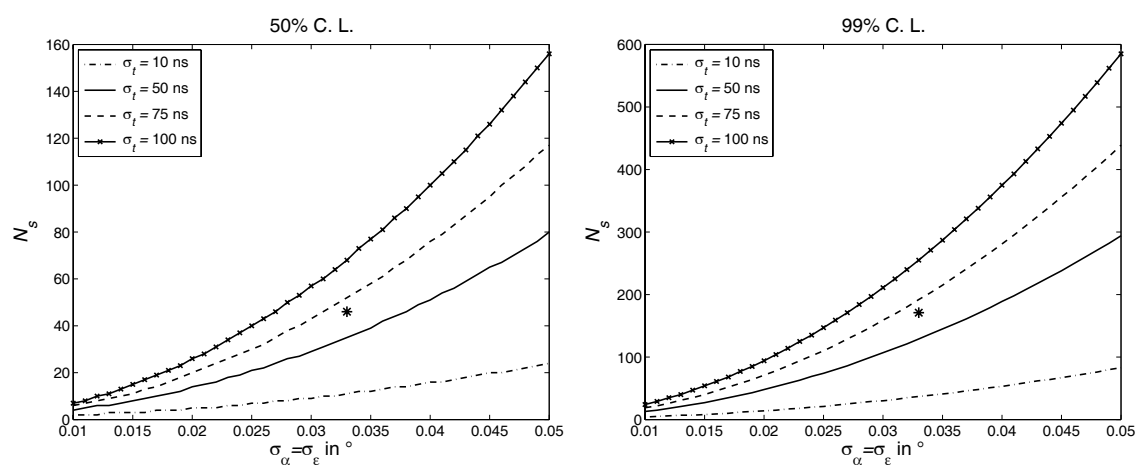

Figure 6: $N_{S}=f\left(\sigma_{\alpha}, \sigma_{t}\right)$ for $50 \%$ and $99 \%$ C. L. (*-MWRL-SWK).

\subsection{Number of rounds}

Calculating the kill probability of $N_{w}$ effective sub-projectiles hitting the target area $A_{T}$, it is assumed that the base area of the fragment cone $A_{E}$ equals the area of the radar CEP $A_{C E P}$ (see fig. 1):

$$
A_{E}(P)=A_{C E P}(P)=\pi\left(R_{R} u \sigma_{\alpha}\right)^{2},
$$

where $R_{R}$ is the distance radar - located grenade and $\sigma_{\alpha}$ is the radar's azimuth deviation assuming $\sigma_{\alpha}=\sigma_{\varepsilon}$. $A_{C E P}$ is valid for a given probability $P$ (via quantile $u$ ) that the grenade is located within this area.

The required probability that at least one out of $N_{w}$ sub-projectiles hits and destructs the target is given by [3]

$$
p_{K, N_{w}}(P)=1-\left(1-p_{K}\right)^{N_{w}(P)},
$$

where $N_{w}(P)=\rho_{f}(P) A_{T}=\frac{N_{f}}{A_{E}(P)} A_{T}$. This leads to the wanted ammunition consumption, i. e. the number of rounds $N_{S}$ to destroy the target with an overallkill probability $P$

$$
N_{S}(P)=\left\lceil\frac{1}{p_{K, N_{w}}(P)}\right\rceil .
$$

\subsection{Simulation results}

This subsection contains the simulation parameters and results to battle an $82 \mathrm{~mm}$ grenade with Ahead ammunition strongly depending on the radar errors. The battle distance is supposed to be $1000 \mathrm{~m}$. Estimating the velocity of an Ahead subprojectile at this point and considering the slowest trajectory velocity of an $82 \mathrm{~mm}$ shell fired on maximum distance, the internal energy $E_{i}$ is $1580 \mathrm{~J}$. Hence, the destruction probability $p_{k \mid H}$ is $74.4 \%$. 
Table 3: Simulation results for MWRL-SWK radar.

\begin{tabular}{|c||c|c|c|c|c|c|}
\hline C. L. in $\%$ & $A_{C E P}$ in $\mathrm{m}^{2}$ & $h_{K}$ in $\mathrm{m}$ & $p_{K}$ in $\%$ & $\rho_{f}$ in $1 / \mathrm{m}^{2}$ & $N_{w}$ & $N_{S}$ \\
\hline \hline 50 & 0.47 & 2.1 & 1.3 & 321 & 1.7 & 46 \\
\hline 90 & 2.82 & 5.2 & 3.2 & 54 & 0.3 & 110 \\
\hline 99 & 6.91 & 8.1 & 5.0 & 22 & 0.1 & 171 \\
\hline
\end{tabular}

In addition to the parameters already given, the quantities are as follows:

$$
l_{M}=329 \mathrm{~mm}, \sigma_{b}=0.3 \mathrm{mil}=0.0169 \mathrm{deg}, \sigma_{t G}=2 \mathrm{~ms}, R_{R}=1000 \mathrm{~m} .
$$

For several overall-kill probabilities $P$, the results are shown in fig. 6 and in tab. 3. They reveal that even with a precise radar like MWRL-SWK an ammunition consumption $N_{S}<100$ can be obtained only for probabilities $P \ll 90 \%$. Furthermore, the distance of the point of ignition to the impact point needs to be short enough such that the sub-projectile's kinetic energy exceeds the minimum energy of $E_{\min }=1160 \mathrm{~J}$. This condition is not fulfilled for $P=99 \%$ in tab. 3 .

\section{Conclusions}

The protection of military camps underlying the threat of attacks by mortar grenades is studied by implementing an early warning system and an air defense system to destroy an approaching shell. The basic mathematical principles are given in order to evaluate or design sensors and ammunition with preformed or natural fragments as well. The study yielded the following most relevant results:

- Determining the $C E P=f\left(\sigma_{\alpha}, \sigma_{t}\right)$ in order to define the edge length of plane warning squares in the military camp, the sensor precision is the bottleneck. Even a very precise radar system like MWRL-SWK $\left(\sigma_{\alpha}=2^{\prime}\right.$, $\sigma_{R}=10 \mathrm{~m}$ ) provides edge lengths of almost $50 \mathrm{~m}$ at $50 \%$ and $100 \mathrm{~m}$ at $99 \%$ C. L. This is impractical considering warning times in a scale of $10 \mathrm{~s}$.

- Therefore, a frontal attack of $82 \mathrm{~mm}$ mortar grenades with a cloud of 152 sub-projectiles originated from $35 \mathrm{~mm}$ Ahead ammunition is investigated. However, the ammunition consumption at $99 \%$ C. L. amounts to 171 shots. The low initial velocity of the sub-projectiles associated with a small mass, a strongly limited number of sub-projectiles, and a very small cone angle $\beta$ of approximately 10 deg restricts the kinetic energy and the hit probability severely. For these reasons, the Ahead ammunition is hardly suited for this application.

- The artillery radar COBRA is completely inappropriate as sensor for both supposed systems.

Considering decisive follow-on tasks in this field, the application of largecaliber HE projectiles instead of Ahead ammunition shall be investigated. In 
addition, the complex combat procedure with detection, tracking, fire control, and interception should be simulated for different threat scenarios.

\section{References}

[1] Athen, H., Ballistik. Quelle \& Meyer: Heidelberg, Germany, 1958.

[2] Gilles, J.T., Computation of the Circular Error Probability Integral. Systems Reseach Center, University of Maryland, 1991.

[3] Germershausen, R., Waffentechnisches Taschenbuch. Rheinmetall GmbH: Duesseldorf, Germany, 1977. 